\title{
Colorimetric Temperature Measurement Method Considering Influence of Ambient Temperature
}

\author{
Bin Zheng ${ }^{1, *}$ and Guoqing Ding ${ }^{1}$ \\ ${ }^{1}$ Instrument Science and technology Department, Shanghai Jiao Tong University, Shanghai, China \\ Corresponding Email: zhengbin22@sjtu.edu.cn
}

\begin{abstract}
Colorimetric temperature measurement has important applications in industrial production. However, it's usually applied to high temperature measurement because it lacks accuracy in medium-low temperature measurement. Aimed at improving the low accuracy of traditional colorimetric temperature measurement method in medium-low temperature measurement, this paper pays attention to the influence of ambient temperature on colorimetric temperature measurement method, especially focusing on the influence of ambient temperature on the measured target and the non-refrigerated infrared detector, and improves the traditional method by considering the influence of ambient temperature. Experimental results show that in the experimental temperature range, the relative error of the method proposed in this paper is less than $1.2 \%$, while the relative error of traditional method is less than $4.0 \%$. So this method can significantly improve the precision of temperature measurement especially in medium-low temperature measurement.
\end{abstract}

\section{Introduction}

Temperature measurement has important applications in industrial production. Temperature measurement can be divided into contact and non-contact measurement. In non-contact measurement, infrared temperature measurement is one of the most important methods. In the application of infrared temperature measurement, there are four methods, including radiation measurement, monochromatic measurement, colorimetric measurement and multi spectrum measurement. Among them, colorimetric temperature measurement method has all the advantages of radiation measurement and monochromatic measurement. What's more, colorimetric temperature measurement can work without knowing the emissivity of the measured target, which avoids the difficulty of emissivity measurement of the target $[1,2]$.

Colorimetric temperature measurement method is usually applied to high temperature measurement(over 600 degree) $[3,4]$. However, it lacks accuracy in medium-low temperature measurement because the influence of ambient temperature should also be considered. This paper discusses the basic principle of colorimetric temperature measurement, then analyses the influence of ambient temperature on the target temperature and infrared detector, and how traditional colorimetric measurement method of calibration and measurement method is improved considering the influence of ambient temperature. Experiments show that compared to traditional method, this method obtains higher accuracy especially in a medium-low temperature measurement.

\section{The mathematical model of the traditional colorimetric temperature measurement method}

In order to reduce the influence of the emissivity of measured target, colorimetric temperature measurement method is put forward. It's based on the measurement of two given wavelength $\lambda_{1}$ and $\lambda_{2}$, and then calculates the radiation power ratio under the two wavelength. In this way, the temperature of the object can be calculated, while the measured temperature is called colorimetric temperature [5].

Colorimetric temperature measurement system has two channels, the output signals are corresponding to the radiation signals of the two channels, which can be expressed as:

$$
V_{1}=K_{1} \varepsilon_{1} \lambda_{1}{ }^{-5} e^{-C_{2} / \lambda_{1} T}, V_{2}=K_{2} \varepsilon_{2} \lambda_{2}^{-5} e^{-C_{2} / \lambda_{2} T}
$$

In Eq. $1, K_{1}$ and $K_{2}$ respectively represent the characteristic constants of the two channels.

The ratio of the outputs of the two channel is:

$$
R=V_{1} / V_{2}=K_{1} \varepsilon_{1} \lambda_{1}^{-5} e^{-C_{2} / \lambda_{1} T} /\left(K_{2} \varepsilon_{2} \lambda_{2}^{-5} e^{-C_{2} / \lambda_{2} T}\right)
$$


From Eq. 2 it can be known that if the ratio $R$ can be calculated, the temperature of the object $T$ can be calculated too. When using this method to measure the temperature of blackbody $T_{S}$, the ratio of the outputs of two channels is:

$$
R=V_{1} / V_{2}=K_{1} \varepsilon_{1} \lambda_{1}^{-5} e^{-C_{2} / \lambda_{1} T_{S}} /\left(K_{2} \varepsilon_{2} \lambda_{2}^{-5} e^{-C_{2} / \lambda_{2} T_{S}}\right)
$$

According to the definition of colorimetric temperature, the relationship between colorimetric temperature and real temperature of the measured target can be expressed as:

$$
1 / T-1 / T_{S}=\lambda_{1} \lambda_{2}\left(\ln \varepsilon_{2}-\ln \varepsilon_{1}\right) /\left[C_{2}\left(\lambda_{2}-\lambda_{1}\right)\right]
$$

3 Measurement considering ambient temperature

There are two kinds of methods to solve temperature drift in the infrared temperature measurement, one method is to change the circuits of the instrument, reducing the influence of ambient temperature on the system. Another method is software compensation, which studies the relationship between the output signal and measured temperature. However, no matter which method is chosen, the system still lacks accuracy especially in medium-low temperature. Therefore we need to further study the relationship between ambient temperature and the measured target temperature. The effect of ambient temperature can be divided into the temperature of external environment, the temperature of infrared detector, the temperature of infrared lens, the temperature of circuit board. Among them, ambient temperature and the temperature of infrared detector are the most important.

Other articles mainly consider the influence of ambient temperature on the target temperature. In fact, in industrial measurement, in order to reduce cost, non-refrigerated infrared detector is commonly used. In this situation, the influence of ambient temperature on non-refrigerating infrared detector should also be considered. In this paper, from these two aspects, the effect of ambient temperature on colorimetric temperature measurement method is analysed in detail.

\subsection{The influence of ambient temperature on the target temperature measurement}

Firstly, establish the mathematical model between target temperature and ambient temperature.

The environment around the measured target has great influence to colorimetric temperature measurement. If measured target temperature is $T_{1}$, ambient temperature is $T_{2}$, the radiation emitted per unit area is $A \varepsilon \sigma T_{1}^{4}$, the radiation absorbed per unit area is $A \varepsilon \sigma T_{2}{ }^{4}$, then the net radiation emitted by objects $Q$ is:

$$
Q=A \varepsilon \sigma T_{1}^{4}-A \alpha \sigma T_{2}^{4}
$$

In Eq. 5, $A$ : unit area; $\varepsilon$ : emissivity of the measured target; $\alpha$ : absorptivity of the measured target. If $\varepsilon=\alpha$, then $Q$ is:

$$
Q=A \varepsilon \sigma\left(T_{1}^{4}-T_{2}^{4}\right)
$$

Table 1 shows one example of energy error of a traditional colorimetric temperature measurement device(wavelength ranges from 9-12 $\mu \mathrm{m})[6]$.

Table 1. The varying relationship of the energy error according to the target temperature and the ambient temperature $\%$.

\begin{tabular}{llllllll}
\hline target temperature & \multicolumn{9}{l}{ ambient temperature $T_{2} / \mathrm{K}$} & & & \\
\cline { 2 - 8 }$T_{1} / \mathrm{K}$ & 270 & 280 & 290 & 300 & 310 & 320 & 330 \\
\hline 500 & 3.64 & 4.35 & 5.12 & 5.96 & 6.86 & 7.82 & 8.83 \\
600 & 2.23 & 2.66 & 3.15 & 3.68 & 4.25 & 4.86 & 5.52 \\
700 & 1.53 & 1.84 & 2.17 & 2.54 & 2.94 & 3.37 & 3.84 \\
800 & 1.14 & 1.37 & 1.62 & 1.90 & 2.20 & 2.53 & 2.88 \\
900 & 0.93 & 1.12 & 1.33 & 1.55 & 1.80 & 2.07 & 2.35 \\
1000 & 0.75 & 0.90 & 1.07 & 1.25 & 1.45 & 1.66 & 1.90 \\
\hline
\end{tabular}

\subsection{The influence of ambient temperature on the non-refrigerated infrared detector}

\subsubsection{Voltage-current characteristic of InGaAs PIN photodiode}

Since the price of non-refrigerating infrared detector is much lower than that of the refrigerated infrared detector, nonrefrigerated infrared detector is widely used in industrial measurement, so the influence of ambient temperature on infrared detector should be analysed. Here takes one of the most frequently used infrared detector InGaAs PIN photodiode for example. 


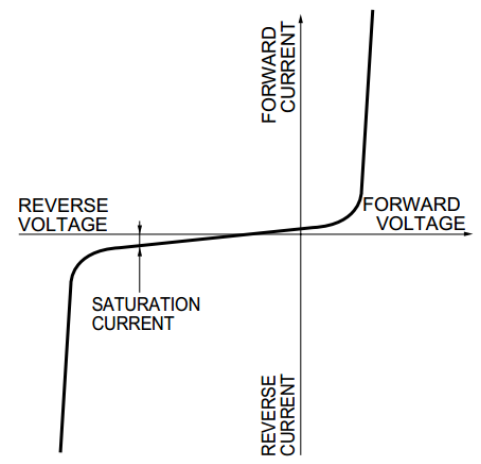

(a) In darkness

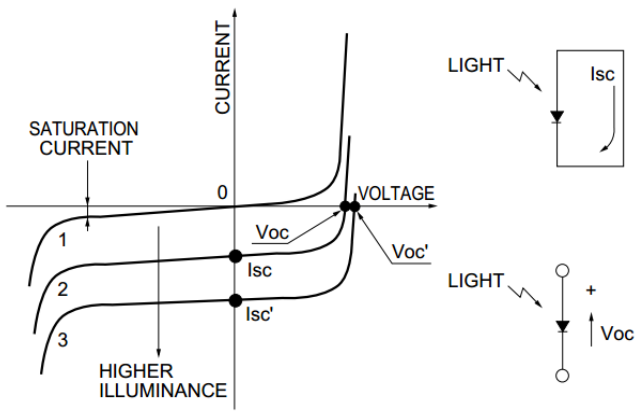

(b) Illuminated

Fig. 1. Voltage-current characteristics.

Figure 1 shows how light can be measured by measuring the photoelectric current. InGaAs PIN photodiode works as follows. When a voltage is applied to an InGaAs PIN photodiode in darkness, a voltage-current characteristic like that shown in Figure 1(a) is obtained. When incident light enters the InGaAs PIN photodiode, the voltage-current characteristic curve becomes as shown in 2 of Figure 1(b). When much stronger incident light enters, the curve shifts as shown in 3 of Figure 1(b). Here, when both terminals of the photodiode are left open, a forward voltage $V_{o p}$ appears, and when both terminals are shorted, a current of $I_{s c}$ flows in the reverse direction.

\subsubsection{Equivalent circuit of InGaAs PIN photodiode}

The equivalent circuit of an InGaAs PIN photodiode is shown in Figure 2. From this equivalent circuit, the short circuit current $I_{S C}$ is given by Eq. 7. The limit of $I_{S C}$ linearity is determined by the 2 nd and 3 rd terms of this formula.

$$
I_{S C}=I_{L}-I_{S}\left[\exp \left(q^{*} I_{S C} R_{S} / k T\right)-1\right]-I_{S C}{ }^{*} R_{S} / R_{S h}
$$

In Eq. 7, $q$ : Electron charge; $k$ : Boltzmann constant; $T$ : Absolute temperature of element; $I_{S}$ : Saturation current of photodiode in the reverse direction.

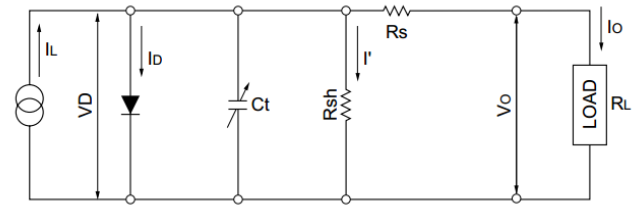

Fig. 2. Equivalent circuit of InGaAs PIN photodiode.

In Figure $2, I_{L}$ : Current generated by incident light(Proportional to the quantity of light); $I_{D}$ : Diode current; $C_{t}$ : Terminal capacitance; $R_{s h}$ : Shunt resistance; $R_{S}$ : Series resistance; $I^{\prime}$ : Shunt resistance current; $V_{D}$ : Voltage across the diode; $I_{O}$ : Output current; $V_{O}$ : Output voltage.

\subsubsection{Temperature characteristics of InGaAs PIN photodiode}




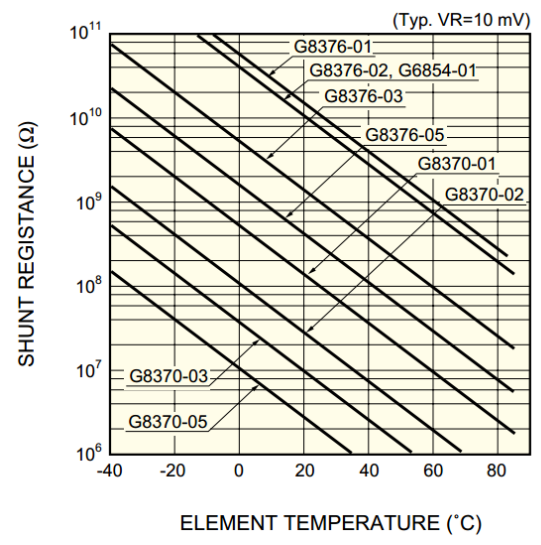

Fig. 3. The impact of element temperature on shunt resistance.

However, spectral response characteristics vary with detector temperature. Temperature characteristics of shunt resistance $R_{s h}$ are shown in Figure 3. Thus, the influence of detector temperature can be analysed.

\section{Experiment}

\subsection{The building of the experiment platform of the colorimetric temperature measurement}

The device needed for experiment mainly consists of blackbody furnace, $24 \mathrm{~V}$ switching power supply and computer. The emissivity of the blackbody furnace HFY-202B is 0.99 and is not an ideal model of 1 . Its accuracy is 1 degree. The computer is used to receive and calculate the experimental data. The environment temperature detector is embedded in the measurement system. In addition, to weaken the influence of other environmental factors as much as possible, experimental environment should be dry and windless.

\subsection{Experimental procedure}

\subsubsection{Calibration experiment of the influence of ambient temperature on infrared detector}

Cover the lens of the temperature measurement system, that is to say, there is no input of infrared radiation. In such situation, put the temperature measurement system input thermostat, record the output of the system in different ambient temperatures in Figure 4

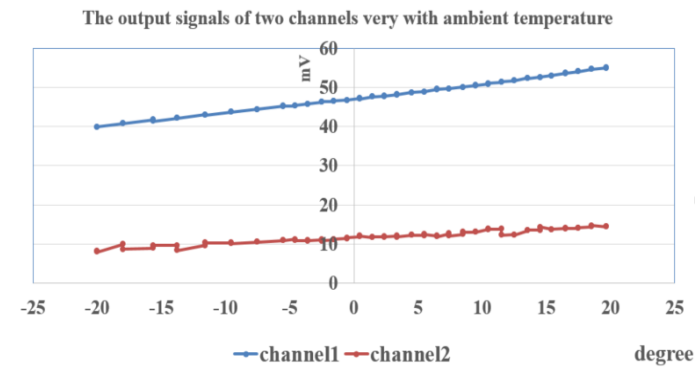

Fig. 4. The output signals of two channels very with ambient temperature.

From Figure 4, it can be concluded that there is linear correlation between the output signal and the temperature of infrared detector.

\subsubsection{Integrated influence of ambient temperature on temperature measurement system}

Since this system cannot be measured in thermostat, that is to say, we cannot individually measure the influence of ambient temperature on target temperature. This experiment measures the system with the influence of ambient temperature both on the measured target and the infrared detector.

Measure the accuracy of the method proposed in this article and traditional method in 10 different temperatures of object being measured. The results are shown in Table 2. 
Table 2. Results of proposed method and traditional method.

\begin{tabular}{ccccc}
\hline Temperature/ degree & $\begin{array}{c}\text { Measured } \\
\text { temperature of } \\
\text { proposed method/ } \\
\text { degree }\end{array}$ & $\begin{array}{c}\text { Relative error of } \\
\text { proposed } \\
\text { method/ } \%\end{array}$ & $\begin{array}{c}\text { Measured } \\
\text { temperature of } \\
\text { traditional method/ } \\
\text { degree }\end{array}$ & $\begin{array}{c}\text { Relative error of } \\
\text { traditional } \\
\text { method } \%\end{array}$ \\
\hline 400 & 395.5 & $1.13 \%$ & 415.7 & $3.93 \%$ \\
420 & 415.9 & $0.98 \%$ & 430.6 & $2.52 \%$ \\
440 & 436.0 & $0.91 \%$ & 451.1 & $2.52 \%$ \\
460 & 455.7 & $0.93 \%$ & 469.4 & $2.04 \%$ \\
480 & 476.1 & $0.81 \%$ & 487.9 & $1.65 \%$ \\
500 & 497.0 & $0.60 \%$ & 507.8 & $1.56 \%$ \\
700 & 603.4 & $0.57 \%$ & 605.4 & $0.90 \%$ \\
800 & 703.9 & $0.56 \%$ & 705.9 & $0.84 \%$ \\
900 & 803.5 & $0.44 \%$ & 805.5 & $0.69 \%$ \\
\hline
\end{tabular}

\subsection{Result analysis}

Table 2 shows the results of the two methods in 10 different temperatures. Usually, temperature more than 600 degree is defined high temperature while temperature lower than 600 degree is called medium-low temperature. In high temperature measurement, the precision of the two methods are relatively high, that is to say, ambient temperature has little effect on high temperature measurement. However, in medium-low temperature measurement, take 400-500 degree for example, for the method proposed in the article, the absolute error is within 4 degree and the relative error is within $1.2 \%$, which still has high accuracy. For the traditional method, the absolute error is within 6 degree and the relative error is within $4 \%$, which lacks accuracy. That is to say, if the influence of ambient temperature discussed in this paper is compensated, the accuracy of colorimetric temperature measurement method can be significantly improved especially in medium-low temperature measurement.

In addition, it can be concluded that with the increase of temperature of object being measured, the influence of the ambient temperature becomes smaller and smaller, which also explains why traditional colorimetric temperature measurement method is suitable for high temperature measurement.

From the above analysis, it can be concluded that the traditional colorimetric temperature measurement method lacks precision for medium-low temperature object measurement. However, the method proposed in the article takes into consideration of the influence of ambient temperature on the measured target and the infrared detector, and compensate the influence of ambient temperature. This method is not only suitable for high temperature target, but also can significantly improve accuracy for medium-low temperature target.

\section{Summary}

Compared to other methods of infrared temperature measurement, traditional colorimetric method can eliminate the effects of emissivity, but ignores the influence of ambient temperature, so it lacks accuracy especially in medium-low temperature measurement. This paper thoroughly analysis the influence of ambient temperature on the measured target and the infrared detector and compensate the errors brought by ambient temperature. Experiments show that this method can significantly improve accuracy for medium-low temperature target, which will be of great significance in practical applications.

\section{References}

1. D. Shi, Xinyang Teachers College, Chinese Journal of Scientific Instrument, 26(12): 1238-1243 (2005)

2. Y. Si, Doctoral dissertation, North University of China (2013)

3. J.H. Zhu, Doctoral dissertation, North University of China (2011)

4. C. Romero, X. Li, S. Keyvan, Applied Thermal Engineering, 25(5-6), 659-676 (2005)

5. P. Sun, Doctoral dissertation, Jilin University (2007)

6. Y. Min, C.W. Peng, Y.H. Yan, Journal of Hunan University, 31(5): 110-112 (2004) 